\title{
Flux Pinning Properties of REBCO coated conductors for High Field Magnets
}

\author{
Satoshi Awaji ${ }^{*}$ and Kazuo Watanabe \\ Institute for Materials Research, Tohoku University
}

Received 13 November 2011; accepted 22 November 2011

\begin{abstract}
From the viewpoint of high field application, the mechanical and critical current properties of recently developed $\mathrm{REBa}_{2} \mathrm{Cu}_{3} \mathrm{O}_{\mathrm{y}}$ (RE123, RE: rare-earth) coated conductors are summarized. In addition, effective flux pinning mechanisms in RE123 are also introduced. As one of the examples for high field application, the upgrading of the $18 \mathrm{~T}$ cryogen-free superconducting magnet is shown. The large anisotropy of $J_{c}$ is a problem at low temperature and high magnetic field. The nanorod is considered as the useful methods to improve the anisotropy of $J_{c}$, although its efficiency becomes small at low temperature.
\end{abstract}

\section{INTRODUCTION}

$\mathrm{REBa}_{2} \mathrm{Cu}_{3} \mathrm{O}_{\mathrm{y}}(\mathrm{RE} 123, \mathrm{RE}=$ rare earth $)$ coated conductors are developed aggressively. Those show not only high in-field critical current density $J_{\mathrm{c}}$ but also high mechanical tolerance. The external stress dependences of critical current density $J_{\mathrm{c}}$ for various practical superconducting wires are summarized in Fig. 1 [1-5]. It is clear that the RE123 coated conductors show the excellent mechanical properties among the practical superconducting wires due to elastic constant and a high yield stress of the Hastelloy substrate. Therefore, RE123 coated conductors on the Hastelloy tape are the promising superconducting wires for high field application. Nowadays, some companies commercially supply the coated conductors produced by their own techniques.

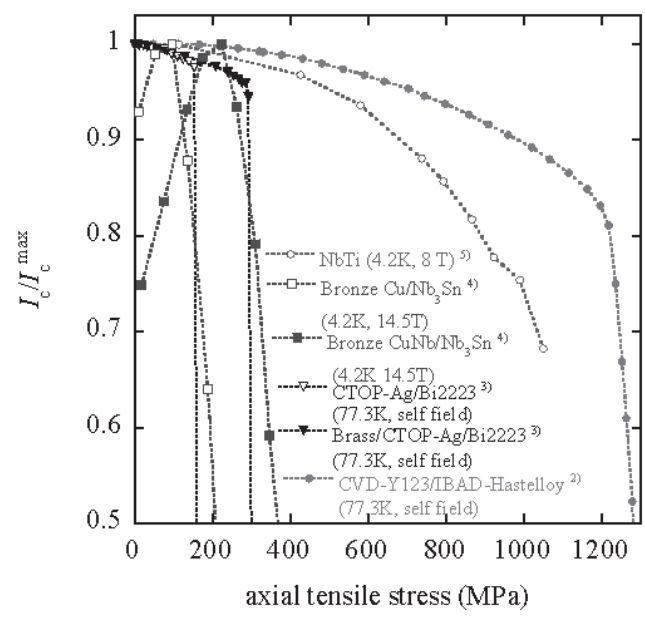

Fig. 1. Stress dependence of $J_{\mathrm{c}}$ for various practical superconducting wires [1-5].

*Corresponding author: awaji@imr.tohoku.ac.jp

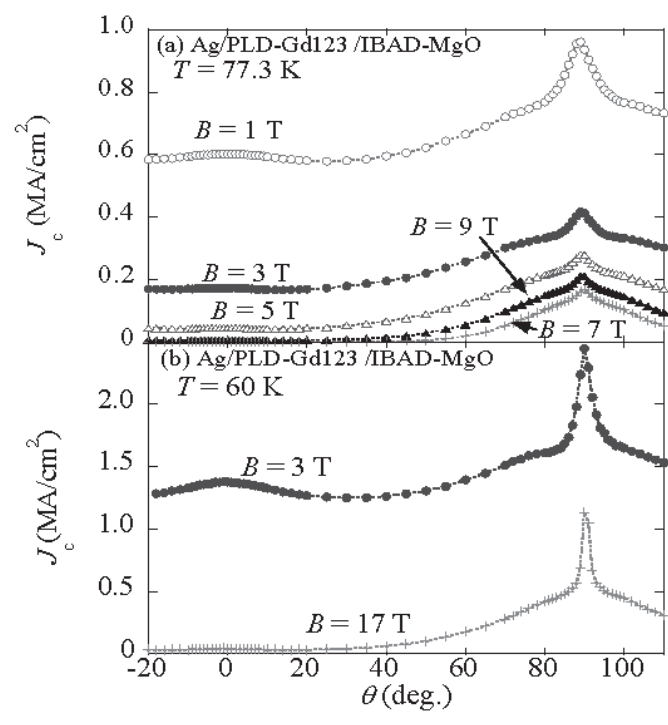

Fig. 2. Angular dependence of $J_{\mathrm{c}}$ at $77.3 \mathrm{~K}$ and $60 \mathrm{~K}$ for PLD-Gd123 coated conductors.

However, the characterizations of $J_{\mathrm{c}}$ on the basis of the flux pinning mechanism are still going on in application-oriented research. In this review, the recent understandings on the flux pinning mechanism in RE123 coated conductors and of high field magnets are introduced.

\section{FLUX PINNING PROPERTY OF COATED CONDUTORS}

\subsection{Intrinsic pinning}

The angular dependence of $J_{\mathrm{c}}$ at 77.3 and $60 \mathrm{~K}$ for PLD-Gd123 coated conductors are shown in Fig. 2. The angular-dependent $J_{\mathrm{c}}$ represents a large sharp peak at $\theta=$ $90^{\circ}(B \perp \mathrm{c})$ and a small broad peak at $\theta=0(B / / \mathrm{c})$. This is a typical behavior for the RE123 films without any artificial pinning centers [6]. In addition, the angular dependence of the $n$-value is qualitatively similar to that of $J_{\mathrm{c}}$ at $77.3 \mathrm{~K}$ as shown in Fig. 3. When the temperature decreases to $60 \mathrm{~K}$, the large $J_{\mathrm{c}}(\theta)$ peak for $B \perp \mathrm{c}$ becomes sharper in comparison with that at $77.3 \mathrm{~K}$. However, the angular dependence of the $n$-value near $B \perp \mathrm{c}$ at $60 \mathrm{~K}$ shows an 


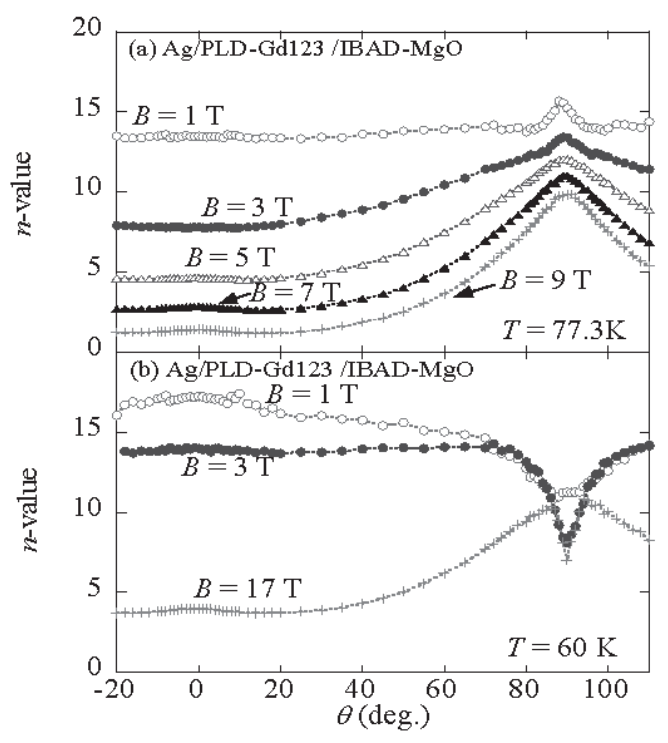

Fig. 3. Angular dependence of $n$-values at $77.3 \mathrm{~K}$ and $60 \mathrm{~K}$ for the PLD-Gd123 coated conductors.

inverse correlation to that of $J_{\mathrm{c}}$. In addition, the $n$-value is as small as about 8 and is independent of the magnetic Fields despite of the large $J_{c}$. It is reported that this behavior is due to the double-kink excitation of the pinned vortices in the periodic correlated pinning centers like the intrinsic pinning [6-8]. If we assume that the inverse angular dependence between $J_{\mathrm{c}}$ and $n$-value is related with the intrinsic pinning, the intrinsic pinning is not effective at $77.3 \mathrm{~K}$ but effective at $60 \mathrm{~K}$. This is quite reasonable because the coherence length along the c-axis becomes large when the temperature is close to the critical temperature [9]. When the intrinsic pinning is effective, however, the small $n$-value for $B \perp \mathrm{c}$, which can be seen in Fig. 3, is a problem for the application of the superconducting magnets. These behaviors were observed in other RE123 coated conductors [6,7]. Figure 4 shows the temperature dependence of $n$-value of the CVD-Y123 coated conductors at $17 \mathrm{~T}$. The $n$-value for $B / / \mathrm{c}$ at $17 \mathrm{~T}$ increase monotonically with decreasing temperature. However, there exists a plateau region on the temperature dependence of $n$-value between 70 and $40 \mathrm{~K}$ for $B \perp \mathrm{c}$ at $17 \mathrm{~T}$, but the $n$-value increase rapidly below $20 \mathrm{~K}$. It is considered that the increase of $n$-values at low temperature associates from the reduction of the thermally induced double kink excitations of the vortices at low temperatures.

At low temperatures, the quite high in-field $J_{\mathrm{c}}$ can be achieved for $B \perp \mathrm{c}$ because of the strong intrinsic pinning. On the other hand, $J_{\mathrm{c}}$ determined by the random pinning for $B / / \mathrm{c}$ is small in comparison with that for $B \perp \mathrm{c}$. In particular, $J_{\mathrm{c}}$ for the intrinsic pinning increases rapidly in comparison with that for the random pinning. Therefore, the anisotropy of $J_{\mathrm{c}}$ becomes larger in higher magnetic field and lower temperatures [7]. In order to overcome the problem of the large anisotropy of $J_{\mathrm{c}}$, the introduction of the nanorod as the c-axis correlated pining is effective as shown in the next section.

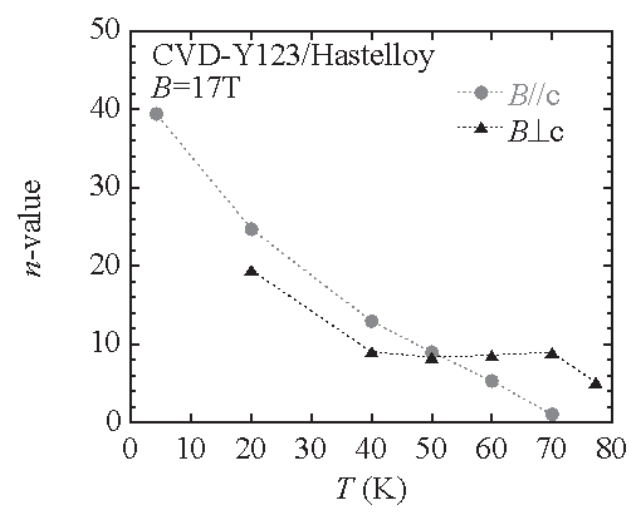

Fig. 4. Temperature dependence of $n$-values at $17 \mathrm{~T}$.

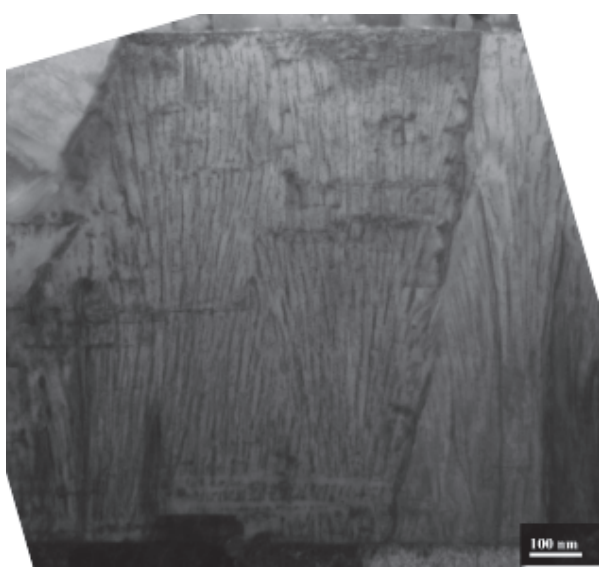

Fig. 5. TEM cross-sectional image of the Zr-doped PLD-Gd123 coated conductors.

\subsection{Nanorod as an artificial pinning center}

Figure 5 shows the TEM cross-sectional image of the Zr-doped Gd123 coated conductor. The nano-scaled rod-shape precipitates can be observed. The nanorods typically align along the c-axis of Gd123 matrix. However those nanorods tend to tilt from the direction of the c-axis of the RE123 matrix with increasing a thickness.

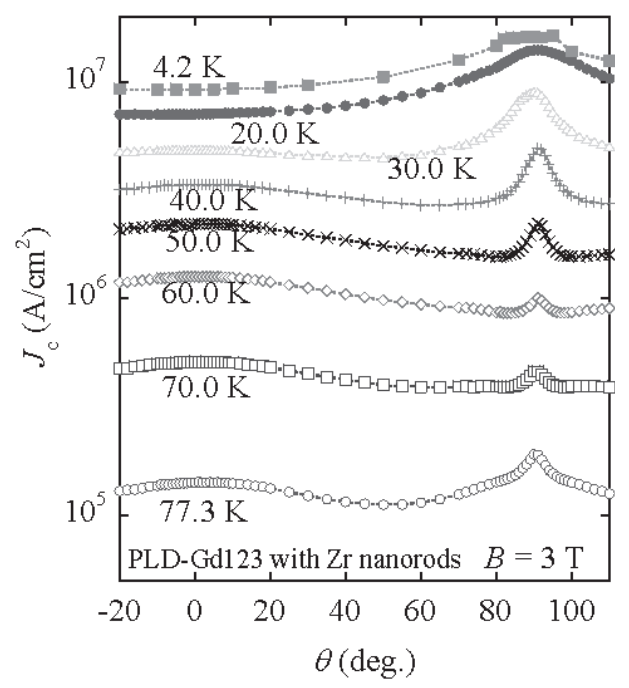

Fig. 6. Angular dependence of $J_{\mathrm{c}}$ for the $\mathrm{Zr}$-doped PLD-Gd123 coated conductors. 


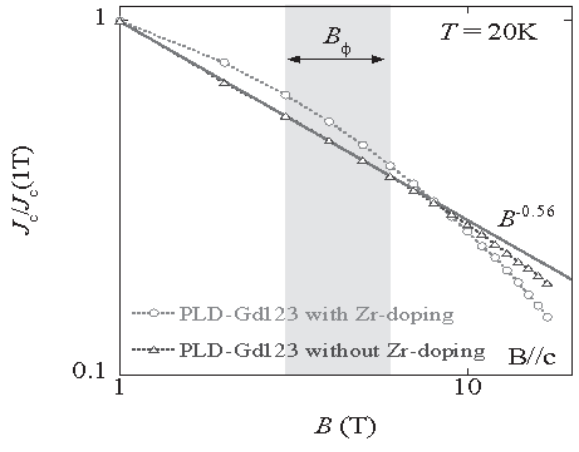

Fig. 7. Field dependence of $J_{c}$ for the $\mathrm{Zr}$-doped and non-doped PLD-Gd123 coated conductors at low temperatures.

It is reported that the nanorods work well as the c-axis correlated pinning center and improve $J_{\mathrm{c}}$ for $\mathrm{B} / \mathrm{c}$ at high temperatures [10]. Figure 6 represents the angular dependence of $J_{c}$ for the Zr-doped PLD-Gd123 coated conductors at $3 \mathrm{~T}$ and at various temperatures. At high temperatures, the large peak of $J_{\mathrm{c}}(\theta)$ for $B / / \mathrm{c}$ appears. This is related to the existence of the $\mathrm{BaZrO}_{3}$ nanorods as shown in Fig. 5. This $J_{\mathrm{c}}(\theta)$ peak along the nanorods increases with decreasing temperature. With further decreasing temperature, however, it decreases after the maximum around $60 \mathrm{~K}$ and disappears at $20 \mathrm{~K}$ at $3 \mathrm{~T}$. The field dependences of $J_{\mathrm{c}}$ for the $\mathrm{Zr}$-doped and undoped Gd123 coated conductors at $20 \mathrm{~K}$, where no peak of $J_{\mathrm{c}}(\theta)$ for $B / / \mathrm{c}$ is observed, are compared each other in Fig. 7. The $J_{\mathrm{c}} \propto B^{-0.5}$ relation can be seen in the low field region below $8 \mathrm{~T}$ for the non-doped Gd123 coated conductor. This means that the random pinning is dominant in the non-doped Gd123. On the other hand, the $J_{\mathrm{c}}$ of the Zr-doped Gd123 coated conductor shows weaker field dependence in the low field region but more steeper field dependence in the high field region. The boundary of the different field dependences of $J_{\mathrm{c}}$ is in agreement with the matching field $B_{\phi} \approx 3-6 \mathrm{~T}$, where $B_{\phi}$ is the magnetic field, at which the density of the magnetic quantum flux is the same as that of the nanorods. Therefore, the flux pinning by the nanorods are still effective at low temperatures, although the contribution of the c-axis correlated pinning against the random pinning becomes small [11]. The cooperation model of the random and correlated pinning centers can explain these phenomena. The detailed discussion is explained in the references [13].

\section{APPLICATION OF COATED CONDUTORS FOR HIGH FIELD MAGVET}

The progress of the superconducting magnets are shown in Fig. 8. The materials used for the innermost coil are also shown in the figure. The magnetic field generated by both of the liquid helium cooled and cryogen-free superconducting magnets increased gradually. The materials for the innermost coil changed with a time and recently high temperature superconducting wires (HTS) are utilized to generate high magnetic field. By the

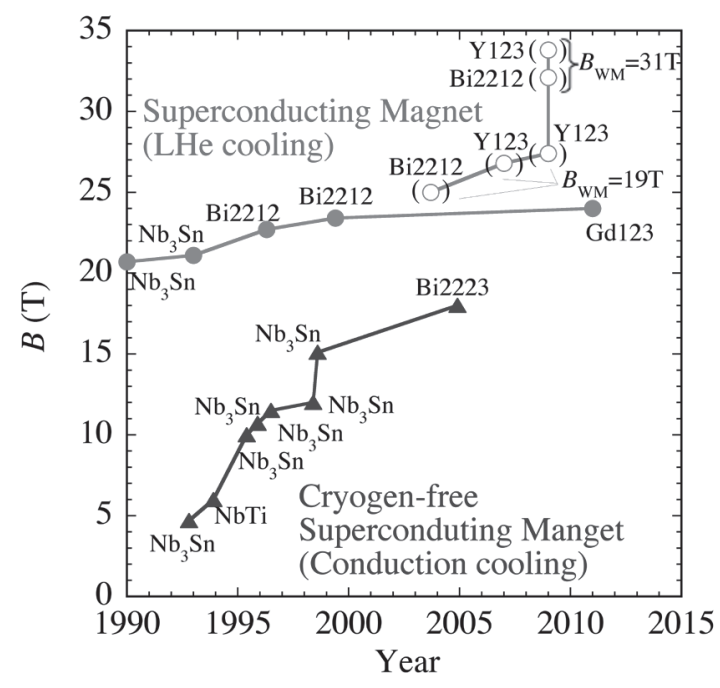

Fig. 8. Progress of liquid He cooled and cryogen-free superconducting magnets.

developments of the long RE123 coated conductors, especially, the very recently in the combination magnet system[14]. The brackets in the figure mean that those are not all superconducting magnets but the small HTS insert coils in the back ground field generated by the resistive or the hybrid magnets. Those test results suggest that the RE123 coils have a capacity of high field generation more than $30 \mathrm{~T}[1,14,15]$. Very recently, the Tsukuba Magnet Laboratory succeeded in the generation of $24 \mathrm{~T}$ by the Gd123 test coil combined with the low temperature superconductor background magnet [15].

The $18 \mathrm{~T}$ cryogen-free superconducting magnet (18T-CSM) at the HFLSM, IMR, Tohoku University consists of an outer $\mathrm{NbTi}$, a middle $\mathrm{Nb}_{3} \mathrm{Sn}$ and an inner $\mathrm{Bi}_{2} \mathrm{Sr}_{2} \mathrm{Ca}_{2} \mathrm{Cu}_{3} \mathrm{O}_{\mathrm{y}}$ (Bi2223) coils as shown in Fig. 9. The $\mathrm{Bi} 2223$ insert coil generates $2.52 \mathrm{~T}$ in a background field of 15.6 $\mathrm{T}$ by the $\mathrm{Nb}_{3} \mathrm{Sn}$ and $\mathrm{NbTi}$ coils [16]. The Ag-alloy sheathed Bi2223 tapes for the inner most coil was reinforced by the co-winding with $0.3 \mathrm{~mm}$ thick stainless

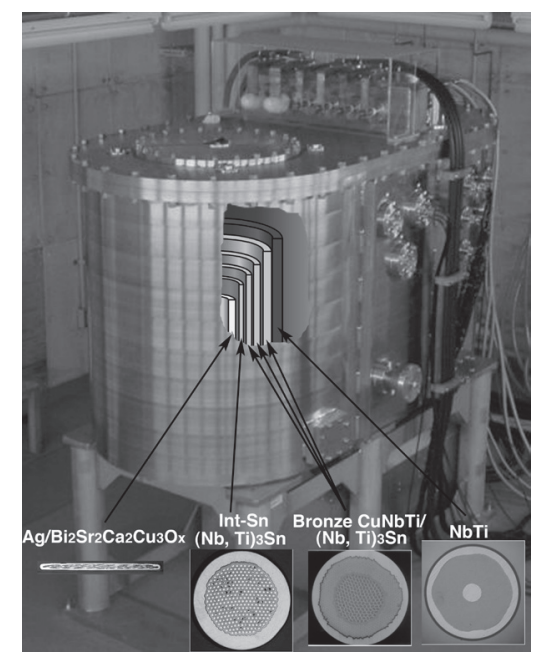

Fig. 9. Picture of the $18 \mathrm{~T}$ cryogen-free superconducting magnet. The cross-sectional images of the wires used in the magnet are demonstrated. 


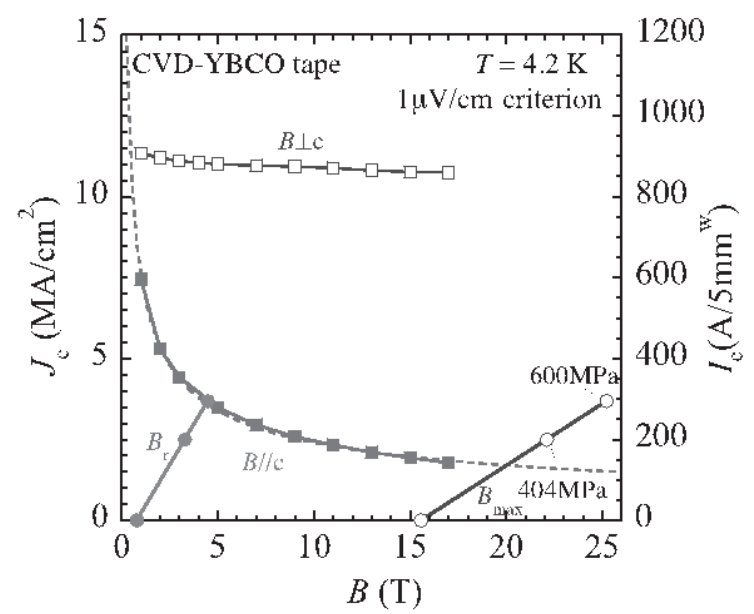

Fig. $10 . I_{\mathrm{c}}$ of the CVD-Y123 coated conductor at $4.2 \mathrm{~K}$ and load lines.

steel tape. If we use the Y123 CC tapes for inner coil, it is expected to achieve higher magnetic field over 20T because of the high mechanical tolerance as show in Fig. 1 $[17,18]$.

We design the size of the Y123 coil similarly to the present Bi2223 insert of the 18T-CSM, in order to replace it. However, the number of turns is increased and the large hoop stress up to $600 \mathrm{MPa}$ is permitted. We found that the central field of 21.9T at $I_{\mathrm{op}}=200 \mathrm{~A}$ for the stress limit of $400 \mathrm{MPa}$ and 25.0 $\mathrm{T}$ at $I_{\mathrm{op}}=295 \mathrm{~A}$ for the stress limit of 600 $\mathrm{MPa}$ can be achieved in the back up field of $15.6 \mathrm{~T}$ generated by the $\mathrm{Nb}_{3} \mathrm{Sn}$ middle and the $\mathrm{NbTi}$ outer coils [17]. Figure 10 represents a load line of the maximum field, $B_{\max }$, together with the $I_{\mathrm{c}}-B$ properties of the CVD-Y123 coated conductors at $4.2 \mathrm{~K}$. The operating current of $295 \mathrm{~A}$ is much smaller than that for $B \perp \mathrm{c}$. In the case of the insert coil, which puts into the large background field, the most magnetic field contribution is parallel to the tape surface, i.e., perpendicular to the c-axis of the Y123 tape. However, we have to care the effects of magnetic field direction, because Y123 has large anisotropy of $J_{\mathrm{c}}$. The radial component of the magnetic field $B_{\mathrm{r}}$, which corresponds to the field parallel to the c-axis, should be compared with $I_{\mathrm{c}}$ for $B / / \mathrm{c}$. In this case, $I_{\mathrm{op}}=295 \mathrm{~A}$ is just on the $I_{\mathrm{c}}-B$ curve for $B / / \mathrm{c}$ as shown in Fig. 10. This means that the $25 \mathrm{~T}$ at $I_{\mathrm{op}}=$ $295 \mathrm{~A}$ is quench point when we use the CVD-Y123 coated conductor [17]. Therefore, the performance of the coated conductor coil is limited by the $I_{\mathrm{c}}$ for $B / / \mathrm{c}$ in this case. The improvement of the anisotropy of $I_{\mathrm{c}}\left(J_{\mathrm{c}}\right)$ is necessary for the development of the high field magnet using the coated conductors. The introduction of the nanorod is one of the good techniques for the improvement of $J_{\mathrm{c}}$ anisotropy as mentioned above.

\section{SUMMARY}

The $J_{\mathrm{c}}$ properties of the RE123 coated conductors are explained on the basis of the flux pinning mechanism. By using $J_{\mathrm{c}}$ properties for the present RE123 coated conductors, the design of the RE123 insert coil is introduced, although the $J_{\text {c }}$ properties of the RE123 coated conductors is still in progress. It is expected that the introduction of the nanorods improves the anisotropy of $J_{\mathrm{c}}$ even at low temperatures and high magnetic fields. The high field superconducting magnets beyond $30 \mathrm{~T}$ will be realize in near future by using the high performance RE123 coated conductors.

\section{ACKNOWLEDGMENTS}

These works were performed with many collaborators. We would like to thank Dr. H. Oguro at the Institute for Materials Research, Tohoku University, Dr. G. Nishijima at the National Institute for Materials Science, Dr. K. Shikimachi and Dr. S. Nagaya at the Chubu Electric Power, Co. Inc., Dr. Izumi and Dr. Y. Shiohara at the ISTEC-SRL, Dr. S. Hanai at the Toshiba Corporation. A part of this work was supported by the New Energy and Industrial Technology Development Organization (NEDO).

\section{REFERENCES}

[1] S. Awaji and K. Watanabe, Oyo Butsuri, vol. 77, pp. 1303-1309, 2008.

[2] M. Sugano, T. Nakamura, K. Shikimachi, N. Hirano, and S. Nagaya, IEEE Trans. Appl. Supercond., vol. 17, pp. 3067-3070, 2007.

[3] K. Osamura, S. Machiya, H. Suzuki, S. Ochiai, H. Adachi, N. Ayai, K. Hayashi and K. Sato, Supercond. Sci. Technol. vol. 21, pp. 054010, 2008.

[4] H. Sakamoto, S. Endoh, Y. Nagasu, K. Wada, A. Kimura, S. Meguro, S. Awaji, K. Watanabe, and M. Motokawa, IEEE Trans. Appl. Supercond., vol. 12, pp. 1067-1070, 2002.

[5] J. Ekin, F. R. Fickett and A. F. Clark, Adv. Cryo. Eng. vol. 22, pp. 449, 1977.

[6] L. Civale, B. Maiorov, J. L. MacManus-Driscoll, H. Wang, T. G. Holesinger, S. R. Foltyn, A. Serquis, and P. N. Arendt: IEEE Trans. Appl. Supercond. vol. 15, pp. 2808-2811, 2005.

[7] S. Awaji, R. Ishihara, K. Watanabe, K. Shikimachi, N. Hirano, S. Nagaya, Appl. Phys. Express, vol. 4, pp. 013101, 2011.

[8] G. Blatter, M. Feilgl'man, V. B. Geshkenbein, A. I. Larkin, and V. Vinokur, Rev. Mod. Phys. vol. 66, pp. 1125-1388, 1994.

[9] S. Awaji, K. Watanabe and N, Kobayashi, Cryogenics, vol. 39, pp. 569-577, 1999.

[10] Y. Yamada, K. Takahashi, H. Kobayashi, M. Konishi, T. Watanabe, A. Ibi, T. Muroga, S. Miyata, T. Kato, T. Hirayama, and Y. Shiohara, Appl. Phys. Lett., vol. 87, pp. 132052, 2005.

[11] S. Awaji, M. Namba, K. Watanabe, S. Ito, E. Aoyagi, H. Kai, M. Mukaida, and S. Okayasu, IEEE Trans. Appl. Supercond. vol. 21, pp. 3192-3195, 2011.

[12] S. Awaji, M. Namba, K. Watanabe, H. Kai, M. Mukaida, S. Okayasu, J. Appl. Phys., submitted.

[13] S. Awaji et al., to be submitted.

[14] H. W. Weijers, U. P. Trociewitz, W. D. Markiewicz, J. Jiang, D. Myers, E. E. Hellstrom, A. Xu, J. Jaroszynski, P. Noyes, Y. Viouchkov, D. C. Larbalestier, IEEE Trans. Appl. Supercond., vol. 20, pp. 576-182, 2010

[15] S. Matsumoto et al., presented at MT22.

[16] G. Nishijima, S. Awaji, S. Hanai and K. Watanabe, Fusion Eng. Design, vol. 81, pp. 2425-2432, 2006.

[17] S. Awaji, R. Ishihara, M. Namba, G. Nishijima, H. Oguro, K. Watanabe, K. Shikimachi, N. Hirano, S. Nagaya, IEEE Trans. Appl. Supercond. vol. 20, pp. 592-595, 2010.

[18] K. Watanabe, S. Awaji, G. Nishijima, S. Hanai, M. Ono, Appl. Phys. Express, vol. 2, pp. 113001, 2009. 